\title{
The mechanism of non-coding RNAs in medulloblastoma (Review)
}

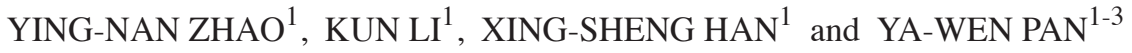 \\ ${ }^{1}$ The Second Medical College of Lanzhou University; ${ }^{2}$ Department of Neurosurgery, \\ Second Hospital of Lanzhou University; ${ }^{3}$ Key Lab of Neurology of Gansu Province, \\ Lanzhou University, Lanzhou, Gansu 730030, P.R. China
}

Received April 20,2021; Accepted August 9, 2021

DOI: $10.3892 / \mathrm{ol} .2021 .13019$

\begin{abstract}
Medulloblastoma (MB) is one of the most common malignant tumors of the central nervous system in children. Although surgery, radiotherapy and chemotherapy have resulted in considerable progress in the treatment of this disease, the prognosis of patients with MB remains very poor. Therefore, highly specific molecular targeted treatment, which can improve the therapeutic efficacy and reduce the side effects of MB, has become a research hotspot. In recent years, non-coding RNAs (ncRNAs), which were initially considered to be transcriptional noise, have been shown to possess regulatory functions. A series of ncRNAs have been identified, including microRNAs and circular RNAs, which affect the expression of specific genes in a variety of tumors. These genes lead to the formation of a specific complex of proteins or they directly participate in protein synthesis in order to regulate the occurrence and development of tumors. The aim of the present review article was to summarize the recent research studies that have explored the ability of ncRNAs to regulate the occurrence and development of MB.
\end{abstract}

\section{Contents}

1. Introduction

2. miRNAs in $\mathrm{MB}$

3. circRNAs in $\mathrm{MB}$

4. Other ncRNAs in MB

5. Conclusions

\section{Introduction}

Medulloblastoma (MB) is a malignant tumor, which exhibits the highest incidence and mortality amongst central nervous

Correspondence to: Dr Ya-Wen Pan, The Second Medical College of Lanzhou University, 222 Tianshui South Road, Chengguan, Lanzhou, Gansu 730030, P.R. China

E-mail: panyawen666@sohu.com

Key words: non-coding RNA, microRNA, circular RNA, medulloblastoma system tumors (1). This disease predominately affects children. According to the 2016 World Health Organization's redefined classification of central nervous system tumors, $\mathrm{MB}$ is divided into four types as follows: Wnt-activated MB, Sonic hedgehog (SHH)-activated MB, group-3 MB and group-4 MB (2), of which, the latter two are the most common types. The prognosis of different subtypes varies considerably. The 5 -year overall survival (OS) rate of Wnt-activated MB can reach 95\%, whereas group-3 exhibits the worst prognosis (45-60\%), and group-4 and SHH-activated MB have an intermediate OS $(75-80 \%)(3,4)$. Since MB is commonly located in the cerebellum, the main symptoms and signs are caused by intracranial hypertension and hydrocephaly, which may be secondary to direct tumor compression or obstruction of cerebrospinal fluid circulation (5). At present, the treatment of MB is primarily based on surgery, radiotherapy and chemotherapy. Although MB is sensitive to radiotherapy and chemotherapy, excessive treatment usually gives rise to serious secondary side effects such as infection, peripheral neuropathy, ototoxicity and myelosuppression in children, who are typically in the developmental stage $(6,7)$. Tumor cells can spread to the spinal cord along the cerebrospinal fluid circulation pathway and $\sim 30 \%$ of patients will develop early tumor spread (8). Concomitantly, since MB exhibits a high degree of malignancy, it commonly relapses after surgery. The efficacy of traditional treatment remains poor. Therefore, the investigation of the molecular mechanism responsible for MB development and the identification of novel therapeutic targets are crucial for the treatment and prevention of this disease.

Approximately $80 \%$ of the genes found in the human genome possess transcriptional activity, whereas only $2 \%$ of the RNA produced by transcription encodes proteins $(9,10)$. The remaining RNA that does not encode proteins is referred to as non-coding RNA (ncRNA) $(9,10)$. Based on their functions, ncRNA molecules are divided into housekeeping RNAs and regulatory RNAs (11). According to the length of the gene, they are divided into short ncRNAs (sncRNAs) and long ncRNAs (lncRNAs). By using $200 \mathrm{nt}$ as the cut-off, sncRNAs may be further subdivided into PIWI-interacting RNAs (piRAN), small nuclear RNAs (snRNA), small nucleolar RNAs (snoRNA) and microRNAs (miRs/miRNAs). Similarly, lncRNAs can be divided into long intergenic non-coding RNAs, natural antisense transcripts, enhancer RNAs (eRNAs), partially unparalleled lncRNAs and circular RNAs (circRNAs), which possess specific structural motifs (Fig. 1) $(12,13)$. At 
present, the involvement of ncRNAs in tumor cell regulation remains unclear. However, numerous studies have investigated various applications based on their functions. For example, miRNAs regulate the stability of transcripts in order to silence genes at the post-transcriptional level (14). circRNAs can be used as miR molecular sponges that participate in adjustment of cell biological function by affecting the functions of miRNAs (15). eRNAs and binding transcription factors form complexes to promote interactions between gene enhancers and promoters (16). In recent years, certain studies have provided significant evidence on the structure and function of ncRNAs. It has been demonstrated that some short ncRNAs, including circRNA, can not only regulate cell function through the aforementioned pathways, but also directly encode various regulatory proteins (17-19). Mounting evidence has shown that ncRNAs can regulate the growth of central nervous system malignant tumors, including gliomas $(11,20,21)$. In addition, it has been shown that the expression levels of various ncRNAs are significantly different between $\mathrm{MB}$ and normal cerebellar cells (22). Therefore, it was hypothesized that deregulated expression of ncRNAs may serve as a marker and/or therapeutic target for MB. Currently, research in this field focuses on the mechanisms of miRNAs has revealed the functions of a large number of miRNAs. However, there are relatively fewer studies on the mechanisms of circRNA and other ncRNAs, which are equally important. This article focuses on the research progress of the role of ncRNAs in MB. In addition, this paper also reviews some of the studies that are expected to be translated into clinical therapeutic and diagnostic targets. The aim of the present article was to review the above content to show the potential of ncRNA in the clinical application of $\mathrm{MB}$.

\section{2. miRNAs in MB}

miRNAs belong to the ncRNA family, which usually includes classes of RNA molecules of 18-25 nucleotides in length. These motifs are highly conserved across different species (11). miRNAs were initially identified in Caenorhabditis elegans in 1993 (23). Since then, research on this topic has been gaining increasing popularity, with a wealth of studies assessing the roles of numerous miRNAs in almost all types of diseases. The current point of view suggests that the main function of miRNAs is to form RNA-induced silencing complexes (RISCs), which include the Argonaute family protein in the cytoplasm (14). RISCs act on the downstream encoding RNAs or ncRNAs to affect cell metabolism at the post-transcriptional level (Fig. 2) (14). It has been shown that the expression levels of specific miRNAs differ on the type and stage of the tumor (24-26). The specific expression patterns of miRNAs determines the development of tumors. Therefore, miRNAs have a wide range of applications for early diagnosis, targeted treatment and prognostic assessment of the tumors.

The analysis of the miRNA expression levels in tissues derived from normal cerebellum and MB has shown similarities with regard to deregulated miRNA expression between $\mathrm{MB}$ and other malignancies (22). The first report that examined the expression of specific miRNAs in MB was performed in 2008 by Pierson et al (27), in which miR-124 expression was predicted to participate in the regulation of the
MB prognostic marker cyclin-dependent kinase 6 (CDK6). Subsequent experiments confirmed that miR-124 expression was decreased in MB and that it affected the expression levels of CKD6 in tumor cells (27). Ferretti et al (28) compared the expression levels of specific miRNAs in normal brain and MB tissues. It was found that miR-18a, miR-19a, miR-21 and miR-25 expression levels in MB were significantly higher than those in normal brain tissues. Concomitantly, the expression levels of the majority of miRNAs, such as miR-9 and miR-125a, were downregulated in tumor samples. These downregulated miRNAs may possess tumor suppressive functions (28). The same is true for cancer and neural stem cells (29). miRNA microarray data analysis from the Gene Expression Omnibus database demonstrated that the expression levels of 22 miRNAs were upregulated in MB, and 26 miRNAs were downregulated (30). It has also been shown that the expression levels of certain miRNAs in MB do not differ from those in normal cerebellum cells. Differential expression of miRNAs has been used as an identification marker of MB molecular subtypes. For example, it was shown that miR-148a expression was specifically enriched in the Wnt-activated MB subtype (31). High expression of the miR-17-92 cluster was observed in SHH-activated MB (32). Zhu et al (33) demonstrated that the expression levels of miR-181a-5p and miR-125b-5p were increased in group-3 MB. However, 12 miRNAs, including miR-18a, miR-135b and miR-660, were overexpressed in group-4 MB (34). These differentially expressed miRNAs were expected to be key indicators for the identification of specific MB-associated markers. Visani et al (35) indicated that there were differences in miR-196B-5P and miR-200B-3P expression between adults and children. Their study suggested that miRNAs may be involved in the development of MB, but not in the differences noted in the biological responses of adults and children with MB. An increasing number of studies have confirmed that the differences in the expression of these miRNAs can affect specific biological processes of MB cells, such as proliferation, migration, invasion and apoptosis. For example, miR-10b is specifically overexpressed in MB cells and it can promote proliferation by mediating the downregulation of the expression of the apoptotic protein Bcl-2 (36). Therefore, miR-10b overexpression can induce apoptosis and inhibit colony formation (36). In SHH-activated MB, the expression of the miR-17-92 cluster regulates N-myc proto-oncogene overexpression (32). Northcott et al (32) confirmed that miR-17-92 clusters can promote proliferation of tumor cells and enhance the invasion of MB cells in vitro. Grunder et al (37) demonstrated that miR-21 overexpression negatively regulated the expression of the transfer inhibitory factor programmed cell death protein 4 in $\mathrm{MB}$ compared with that observed in normal tissues, thereby increasing the expression levels of the downstream invasion medium proteins mitogen-activated protein kinase kinase and $\mathrm{JNK}$, which promote tumor cell invasion. Yang et al (38) analyzed the miRNA expression profiles in 29 patients with $\mathrm{MB}$ and screened this group for miR-192 expression, which was downregulated in tumor cells. The results of their study confirmed that miR-192 inhibited the proliferation and anchorage capability of MB cell lines by regulating the downstream target genes dihydrofolate, integrin $\alpha-\mathrm{V}$ precursor, integrin $\beta-1 / 3$ precursor and cluster of 


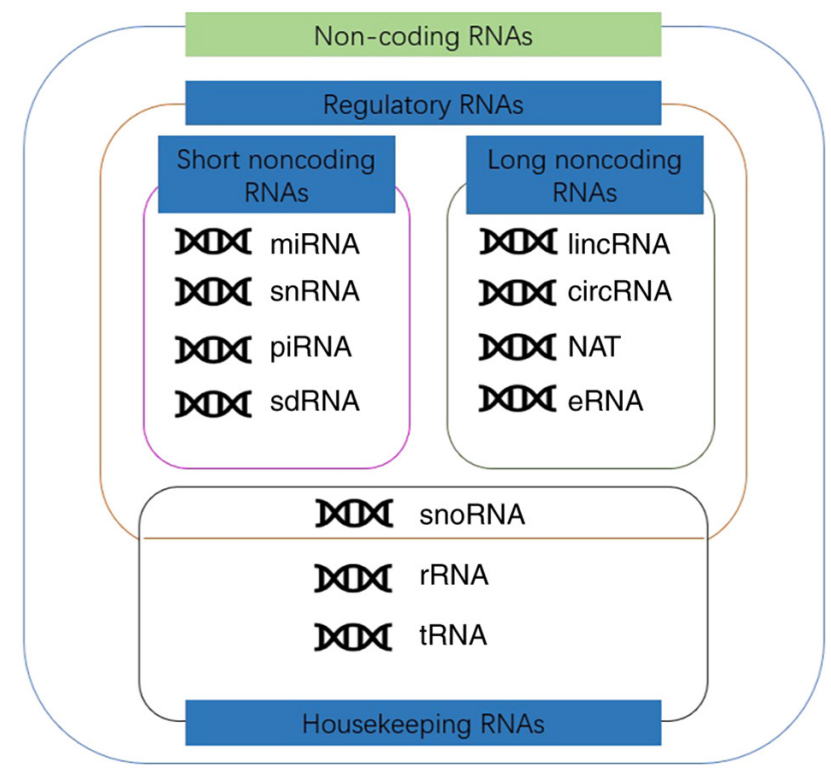

Figure 1. Classification of non-coding RNAs. miRNA, microRNA; snRNA, small nuclear RNA; piRNA, PIWI-interacting RNA; sdRNA, snoRNA derived small RNA; lincRNA, Large intergenic noncoding RNA; circRNA, circular RNA; NAT, natural antisense transcript; eRNA, enhancer RNA; snoRNA, small nucleolar RNA; rRNA, ribosomal RNA; tRNA, transfer RNA.

differentiation 47. This finding was also confirmed in a nude mouse xenograft model, suggesting that miR-192 is a type of metastasis inhibitory factor (38). In MB, the natural antisense transcript HOTAIR of IncRNA HOX competitively binds to miR-1 and miR-206 and causes upregulation of Yin Yang 1 protein expression. This pathway promotes the malignant phenotype of MB (39). Senfter et al (40) demonstrated that the loss of miR-4521 expression led to the activation of the proto-oncogene forkhead box protein M1 (FOXM1) in MB. Kumar et al (41) indicated that miR-217 promoted tumor growth by negatively regulating the target genes sirtuin 1, Roundabout1, FOXO3 and SMAD7. Early diagnosis and prognostic assessment are important steps in the treatment of MB. Currently, the detection of miR expression offers an alternative to imaging data. Li et al (42) demonstrated that miR-449a expression was downregulated in $\mathrm{MB}$ of all subtypes, with the exception of Wnt-MB. This suggests the potential applications of this $\mathrm{miR}$ in the diagnosis of Wnt-MB (42). Pezuk et al (43) examined the expression levels of Polo-like kinase family members and their associated miRNAs (miR-100, miR-126, miR-219 and miR-593) in 32 clinical samples of MB in association with disease prognosis. The results indicated that patients with higher expression of miR-100 and lower expression of miR-126 and miR-219 had improved OS (43). Although increasing evidence has suggested that miR-targeted therapy is of considerable value, miRNA-targeting for the treatment of $\mathrm{MB}$ remains under investigation. Some targeted treatments for specific miRNAs have been shown to inhibit the development of the malignant phenotype of MB cells. For example, overexpression of miR-34a can reduce the expression of transmembrane protein $\delta$-Likel in MB to inhibit tumor cell proliferation and induce apoptosis (44). De Antonellis et al (44) demonstrated inhibition of tumor growth in a nude mouse MB model by administration of adenoviral vectors carrying miR-34a. The minichromosome maintenance protein (MCM2-7) complex has the ability to influence DNA transcription and replication. CDK6, which is part of the MCM2-7 complex, is overexpressed in one-third of MB cases. Therefore, it may be used as a specific marker of disease prognosis. Silber et al (45) established a nude mouse model of heterotopic transplanted tumor with D425 cells, which were infected with lentiviruses containing miR-124. The results indicated that miR-124-targeted CDK6 and effectively inhibited tumor growth (45). However, the development of high-efficiency miR-targeted drugs that can be applied clinically requires further exploration due to the limitations of the current technologies.

\section{3. circRNAs in MB}

In 1991, Nigro et al (46) reported the identification of a new type of RNA, termed circRNA, which was formed by exons. Salzman et al (47), sequenced 15 types of normal or cancerous cells using RNA-seq, including a fetal pulmonary fibroblast line and a leukemia cell line. A total of 80 types of circRNAs were identified (47). Following this study, circRNAs became a research hotspot. It has been shown that circRNAs are widely expressed in eukaryotes. They possess rich, stable, highly conserved and non-randomized motifs (48). Of the annotated circRNAs, $99 \%$ require cutting of the exon at the $3^{\prime}$ and $5^{\prime}$ splicing sites. The majority of the circRNAs contain 2-3 exons. Subsequently, the $3^{\prime}$ end of the clipped segment is spliced with the $5^{\prime}$ end to form a ring. Due to this special circular structure, circRNAs exhibit high stability compared with linear RNAs and are affected to a lesser extent by RNases (49-52). It has also been reported that circRNAs are widely expressed in the brain (49).

Currently, the prevailing hypothesis is that the main role of circRNAs is to combine with corresponding miRNAs through conserved sites. Subsequently, circRNAs act as molecular sponges of miRNAs and regulate various cellular functions (53-58) (Fig. 3). circRNAs are often dysregulated in a variety of malignant tumors, including glioma (59-61). These molecules are involved in regulation of tumor growth, suggesting that they may be important regulators in the development of MB. Lv et al (62) selected 4 pairs of normal cerebellum and $\mathrm{MB}$ tissue samples for gene sequencing and identified 33 differentially expressed circRNAs in MB tissues. The upregulation of two of these circRNAs, which were identified as circular-spindle and kinetochore associated complex subunit 3 (circ-SKA3) and circ-DTL, reverted the malignant phenotype of MB (62). A previous study observed significantly higher expression levels of circ-SKA3 in MB compared with the corresponding expression noted in normal tissues. This was achieved by detecting the expression levels of specific circRNAs that were differentially expressed in MB. The expression levels of the downstream target miR-383-5p were determined by luciferase assay. Subsequent experiments indicated that miR-383-5p expression was influenced by low expression of circ-SKA3 in tumor cells. Following restoration of miR-383-5p expression by silencing circ-SKA3, the expression levels of the downstream FOXM1 protein were also 


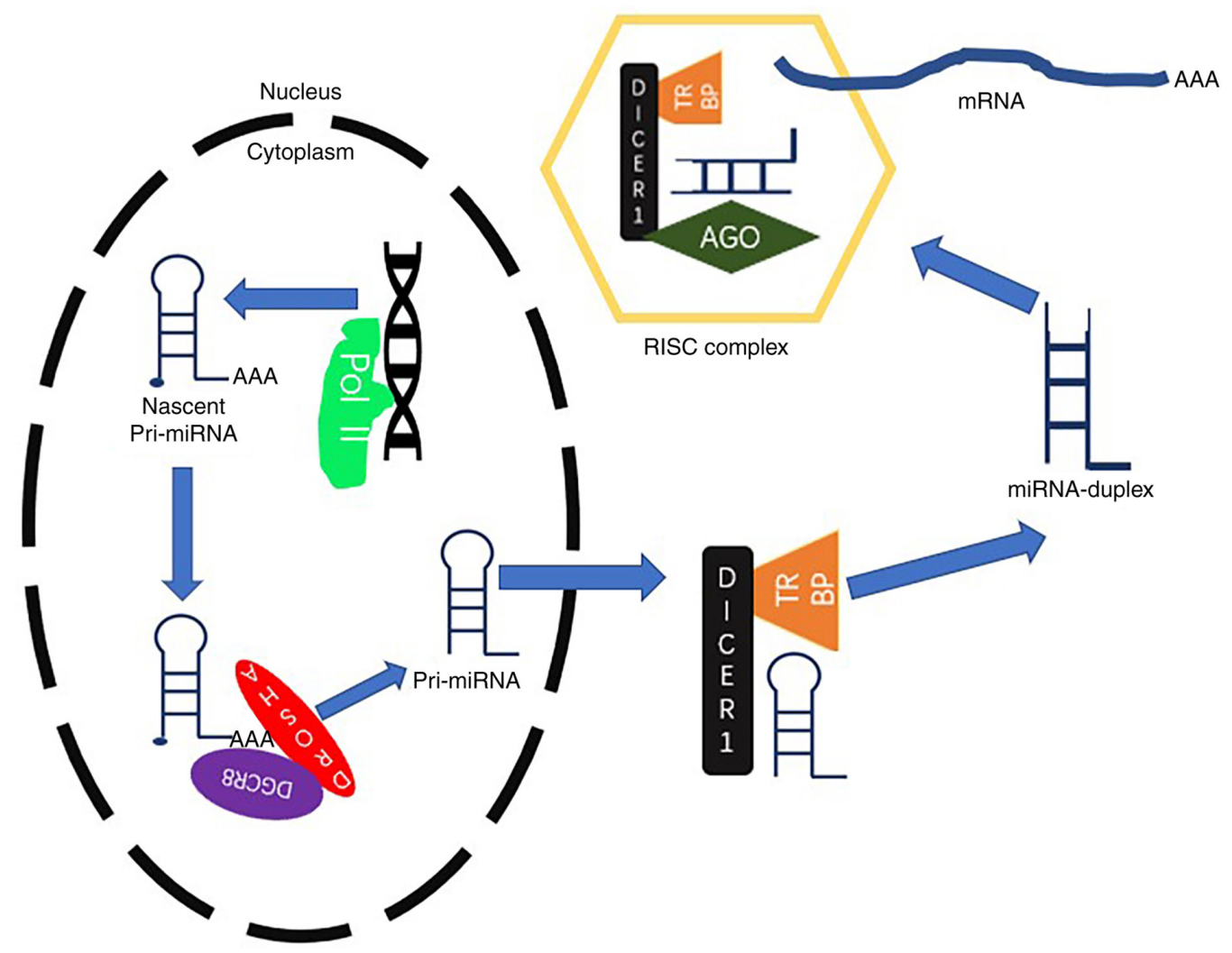

Figure 2. Generation and role of miRNAs: First, nascent pri-miRNAs are generated by related genes mediated by pol II, and then the poly A-tail and m7G-PPNmN cap are removed in the nucleus by shearing of the DROSHA-DGCR8 complex to form precursor miRNAs. After the pri-miRNA translocates to the cytoplasm, DICER1 complex modifies it during a second round of processing and produces miRNA-duplexes containing the mature miRNA strand. Finally, the miRNA binds to the AGO-containing RISC complex to inhibit mRNA function. miRNA, microRNA; pri-miRNA, primary miRNA; RISC, RNA-induced silencing complex; DGCR8, DiGeorge syndrome critical region 8; pol II, RNA polymerase II; TRBP, Tar RNA-binding protein.

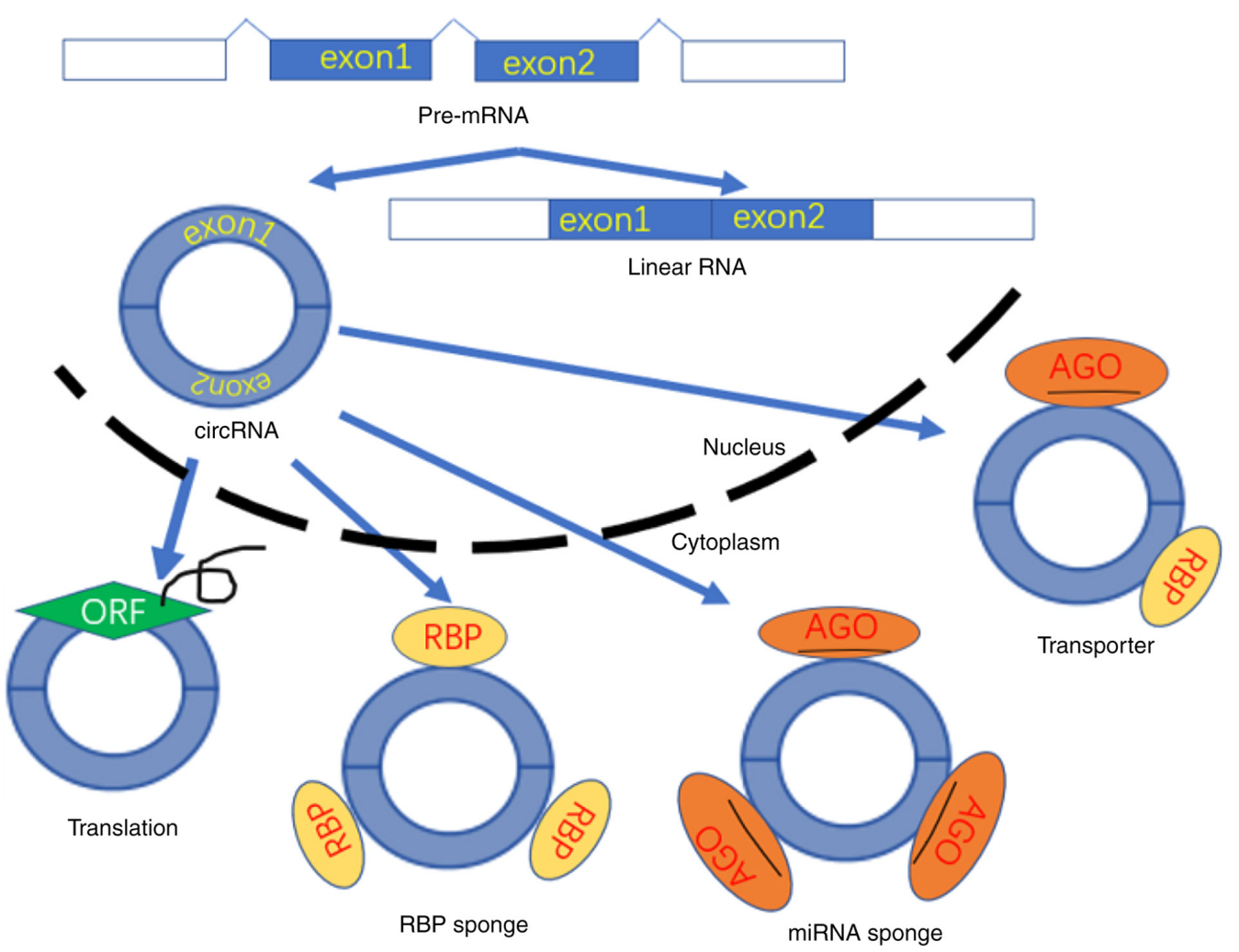

Figure 3. Generation and role of circRNAs: The spliced flanking introns bind to specific sites of the intron either by complementation or by RBPs to form a special ring structure. This then functions as a molecular sponge, or affects RBP and miRNAs in the cytoplasm. Certain circRNAs with an ORF can autonomously generate proteins that participate in regulation. circRNA, circular RNA; ORF, open reading frame; RBP, RNA binding protein. 
affected and the proliferation, migration and invasion of the tumors were inhibited, whereas the induction of apoptosis was enhanced (63). Although current evidence suggests that circRNAs play an important role in the development of $\mathrm{MB}$, additional studies are required to assess their specific mechanisms and potential applications in the clinical treatment of MB.

\section{Other ncRNAs in MB}

At present, the regulatory mechanism of ncRNA expression in MB has been primarily examined by assessing the expression levels of miRNAs and circRNAs. Pertinent ncRNA regulatory functions have not been thoroughly investigated. For example, eRNAs, which were identified relative more recently, play a regulatory role by forming a complex with RNA polymerase II DNA binding transcription factor and the RNA binding transcription factor that binds to the gene enhancer (64). Lin et al (65) investigated the binding of H3K27AC and bromodomain-containing protein 4 by chromatin immunoprecipitation assays in MB tissue matched samples. DNA methylation and transcription data were also provided to describe 28 cis-regulatory elements in MB. The results indicated that the differential regulation of enhancers was heterogeneous between subgroups (65). That is, eRNAs may play an important role in the phenotypic changes of MB. SPRY4 intronic transcript 1 (SPRY4-IT1) is a type of lncRNA with a length of $\sim 706 \mathrm{bp}$. It has a hairpin structure and is expressed in gliomas. Shi et al (66) demonstrated that inhibition of SPRY4-IT1 affected the expression of MMP-2 in MB, and the migration of the MB cell line Daoy was decreased by this pathway.

\section{Conclusions}

$\mathrm{MB}$ is one of the most common types of central nervous system malignant tumors encountered in children, and it is characterized by a high incidence and a poor prognosis. Although significant progress has been made in exploring the development and pathogenesis of MB, the effects of currently available treatments are often unsatisfactory due to the propensity of the tumor for recurrence and metastatic spread, and the occurrence of serious complications. In recent years, it has been confirmed that several ncRNAs, such as miRNAs and circRNAs, are associated with the regulatory mechanisms involved in the occurrence and development of various tumors. It has also been shown that several ncRNAs, including miRNAs and circRNAs, regulate tumor metabolism and are involved in the development of MB. At present, a number of studies have been conducted on the regulatory mechanism of miRNAs in MB. It has been confirmed that miRNAs play an important role in the occurrence and development of MB. However, a limited number of studies have been performed on the mechanisms associated with the expression of other ncRNAs, including circRNAs, and the development of MB. It is considered that wider adoption of high-throughput microarray and second-generation sequencing technologies will enable the full investigation of ncRNA-associated mechanisms and their clinical applications in the diagnosis and treatment of $\mathrm{MB}$.

\section{Acknowledgements}

Not applicable.

\section{Funding}

The present study was supported by grants from the Science and Technology Research Project of Gansu Province (grant nos. 145RTSA012 and 17JR5RA307), the Project of Healty and Family Planing Commission of Gansu (grant no. GSWSKY2014-31/GSWSKY-2015-58/GSWSKY2018-01), the Lanzhou Science and Technology Bureau Project (grant no. 2018-1-109), the Cuiying Science and Technology fund (grant no. CY2017-MS12/CY2017-MS15/CY2017-BJ15/CYXZ-01), Cuiying Graduate Supervisor Applicant Training Program (grant no. 201803) and the Special fund project for doctoral training (grant no. YJS-BD-13) of Lanzhou University Second Hospital.

\section{Availability of data and materials}

Not applicable.

\section{Authors' contributions}

YNZ completed the primary writing and proofreading of the manuscript. KL made partial corrections to the original anuscript and made the figures. XSH performed the literature search. YWP directed the writing of the article and made partial revisions. All authors have read and approved the final manuscript.

\section{Ethics approval and consent to participate}

Not applicable.

\section{Patient consent for publication}

Not applicable.

\section{Competing interests}

The authors declare that they have no competing interests.

\section{References}

1. Ostrom QT, Gino C, Gittleman H, Patil N, Waite K, Kruchko C and Barnholtz-Sloan JS: CBTRUS statistical report: Primary brain and other central nervous system tumors diagnosed in the United States in 2012-2016. Neuro Oncol 21 (Suppl 5): v1-v100, 2019.

2. Louis D, Perry A, Reifenberger G, von Deimling A, FigarellaBranger D, Cavenee WK, Ohgaki H, Wiestler OD, Kleihues P and Ellison DW: The 2016 World Health Organization Classification of Tumors of the Central Nervous System: A summary. Acta Neuropathol 131: 803-820, 2016.

3. Forbes K: Osborn, Salzman, Barkovich, et al: Diagnostic Imaging: Brain. 2nd edition. Neuroradiology 54: 269, 2012.

4. Gajjar A and Robinson G: Medulloblastoma-translating discoveries from the bench to the bedside. Nat Rev Clin Oncol 11: 714-722, 2014.

5. Pollack IF and Jakacki RI: Childhood brain tumors: Epidemiology, current management and future directions. Nat Rev Neurol 7: 495-506, 2011. 
6. Merchant TE, Mulhern RK, Krasin MJ, Kun LE, Williams T, Li C, Xiong X, Khan RB, Lustig RH, Boop FA and Sanford RA: Preliminary results from a phase II trial of conformal radiation therapy and evaluation of radiation-related CNS effects for pediatric patients with localized ependymoma. J Clin Oncol 22: 3156-3162, 2004.

7. Kortmann RD, Kühl J, Timmermann B, Mittler U, Urban C, Budach V, Richter E, Willich N, Flentje M, Berthold F, et al: Postoperative neoadjuvant chemotherapy before radiotherapy as compared to immediate radiotherapy followed by maintenance chemotherapy in the treatment of medulloblastoma in childhood: Results of the german prospective randomized trial hit ' 91 . Int J Radiat Oncol Biol Phys 46: 269-279, 2000.

8. Gerber NU, Mynarek M, Von Hoff K, Friedrich C, Resch A and Rutkowski S: Recent developments and current concepts in medulloblastoma. Cancer Treat Rev 40: 356-365, 2014.

9. ENCODE Project Consortium: An Integrated Encyclopedia of DNA Elements in the Human Genome. Nature 489: 57-74, 2012.

10. Pennisi E: ENCODE project writes eulogy for junk DNA. Science 337: 1159, 1161, 2012.

11. Ramasamy P, Malhotra M and Massoud TF: The protean world of non-coding RNAs in glioblastoma. J Mol Med (Berl) 97: 909-925, 2019 .

12. Nie L, Wu HJ, Hsu JM, Chang SS, Labaff AM, Li CW, Wang Y, Hsu JL and Hung MC: Long non-coding RNAs: Versatile master regulators of gene expression and crucial players in cancer. Am J Transl Res 4: 127-150, 2012.

13. Barrett SP and Salzman J: Circular RNAs: Analysis, expression and potential functions. Development 143: 1838-1847, 2016.

14. Peng Y and Croce CM: The role of MicroRNAs in human cancer. Signal Transduct Target Ther 1: 15004, 2016.

15. Kristensen LS, Hansen TB, Ven $\varnothing$ MT and Kjems J: Circular RNAs in cancer: Opportunities and challenges in the field. Oncogene 37: 555-565, 2018.

16. Trimarchi T, Bilal E, Ntziachristos P, Fabbri G, Dalla-Favera R, Tsirigos A and Aifantis I: Genome-wide mapping and characterization of Notch-regulated long noncoding RNAs in acute leukemia. Cell 158: 593-606, 2014.

17. Xia X, Li X, Li F, Wu X, Zhang M, Zhou H, Huang N, Yang X, Xiao F, Liu D, et al: A novel tumor suppressor protein encoded by circular AKT3 RNA inhibits glioblastoma tumorigenicity by competing with active phosphoinositide-dependent Kinase-1. Mol Cancer 18: 131, 2019.

18. Gao X, Xia X, Li F, Zhang M, Zhou H, Wu X, Zhong J, Zhao Z, Zhao K, Liu D, et al: Circular RNA-encoded oncogenic E-cadherin variant promotes glioblastoma tumorigenicity through activation of EGFR-STAT3 signalling. Nat Cell Biol 23: 278-291, 2021

19. Morlando M, Di Timoteo G, Rossi F, Morlando M, Briganti F, Sthandier O, Fatica A, Santini T, Andronache A, Wade M, et al: Circ-ZNF609 is a circular RNA that Can Be translated and functions in myogenesis. Mol Cell 66: 22-37.e9, 2017.

20. Diederichs S: Non-coding RNA in malignant tumors. A new world of tumor biomarkers and target structures in cancer cells. Pathologe 31 (Suppl 2): S258-S262, 2010 (In German).

21. Julia L, Grabowska A, Zarebska Ż, Kuczyński K, Kuczyńska B and Rolle K: Non-coding RNAs in Brain Tumors, the Contribution of lncRNAs, circRNAs, and snoRNAs to cancer development-their diagnostic and therapeutic potential. Int J Mol Scie 21: 7001, 2020.

22. Joshi P, Katsushima K, Zhou R, Meoded A, Stapleton S, Jallo G, Raabe E, Eberhart CG and Perera RJ: The therapeutic and diagnostic potential of regulatory noncoding RNAs in medulloblastoma. Neurooncol Adv 1: vdz023, 2019.

23. Lee RC, Feinbaum RL and Ambros V: The C. elegans Heterochronic gene lin-4 encodes small RNAs with antisense complementarity to lin-14. Cell 75: 843-854, 1993.

24. Browne BM, Stensland KD, Patel CK, Sullivan T, Burks EJ, Canes D, Raman JD, Warrick J and Reiger-Christ KM: MicroRNA expression profiles in upper tract urothelial carcinoma differentiate tumor grade, stage, and survival: Implications for clinical decision-making. Urology 123: 93-100, 2019.

25. Magdalena Z, Fendler W, Zakrzewski K, Sikorska B, Grajkowska W, Dembowska-Bagińska B, Filipek I, Stefańczyk Ł and Liberski PP: Altered MicroRNA expression is associated with tumor grade, molecular background and outcome in childhood infratentorial ependymoma. PLoS One 11: e0158464, 2016.

26. Rothé F, Ignatiadis M, Chaboteaux C, Haibe-Kains B Kheddoumi N, Majjaj S, Badran B, Fayyad-Kazan H, Desmedt C, Harris AL, et al: Global microRNA expression profiling identifies MiR-210 associated with tumor proliferation, invasion and poor clinical outcome in breast cancer. PLoS One 6: e20980, 2011.
27. Pierson J, Hostager B, Fan R and Vibhakar R: Regulation of cyclin dependent kinase 6 by microRNA 124 in medulloblastoma. J Neurooncol 90: 1-7, 2008.

28. Ferretti E, De Smaele E, Po A, Di Marcotullio L, Tosi E, Espinola MS, Di Rocco C, Riccardi R, Giangaspero F, Farcomeni A, et al: MicroRNA profiling in human medulloblastoma. Int J Cancer 124: 568-577, 2010.

29. Po A, Abballe L, Sabato C, Gianno F, Chiacchiarini M, Catanzaro G, De Smaele E, Giangaspero F, Ferretti E, Miele E and Besharat ZM: Sonic hedgehog medulloblastoma cancer stem cells mirnome and transcriptome highlight novel Functional Networks. Int J Mol Sci 19: 2326, 2018.

30. Dai J, Li Q, Bing Z, Zhang Y, Niu L, Yin H, Yuan G and Pan Y: Comprehensive analysis of a microRNA expression profile in pediatric medulloblastoma. Mol Med Rep 15: 4109-4115, 2017.

31. Yogi K, Sridhar E, Goel N, Jalali R, Goel A, Moiyadi A, Thorat R, Panwalkar P, Khire A, Dasgupta A, et al: MiR-148a, a microRNA upregulated in the WNT subgroup tumors, inhibits invasion and tumorigenic potential of medulloblastoma cells by targeting Neuropilin 1. Oncoscience 2: 334-348, 2015

32. Northcott PA, Fernandez-L A, Hagan JP, Ellison DW, Grajkowska W, Gillespie Y, Grundy R, Van Meter T, Rutka JT, Croce CM, et al: The miR-17/92 polycistron is up-regulated in sonic hedgehog-driven medulloblastomas and induced by $\mathrm{N}$-myc in sonic hedgehog-treated cerebellar neural precursors. Cancer Res 69: 3249-3255, 2009.

33. Zhu LY, Wu XY, Liu XD, Zheng DF, Li HS, Yang B, Zhang J and Chang Q: Aggressive medulloblastoma-derived exosomal miRNAs promote in vitro invasion and migration of tumor cells via Ras/MAPK pathway. J Neuropathol Exp Neurol 79: 734-745, 2020.

34. Gershanov S, Toledano H, Michowiz S, Barinfeld O, Pinhasov A, Goldenberg-Cohen N and Salmon-Divon M: MicroRNA-mRNA expression profiles associated with medulloblastoma subgroup 4. Cancer Manag Res 10: 339-352, 2018.

35. Visani M, Marucci G, Biase D, Giangaspero F, Buttarelli FR, Brandes AA, Franceschi E, Acquaviva G, Ciarrocchi A, Rhoden KJ, et al: miR-196B-5P and miR-200B-3P are differentially expressed in medulloblastomas of adults and children. Diagnostics (Basel) 10: 265, 2020.

36. Pal R and Greene S: microRNA-10b is overexpressed and critical for cell survival and proliferation in medulloblastoma. PLoS One 10: e0137845, 2015.

37. Grunder E, D'ambrosio R, Fiaschetti G, Abela L, Arcaro A, Zuzak T, Ohgaki H, Lv SQ, Shalaby $\mathrm{T}$ and Grotzer M: MicroRNA-21 suppression impedes medulloblastoma cell migration. Eur J Cancer 47: 2479-2490, 2011.

38. Yang SY, Choi SA, Lee JY, Park AK, Wang KC, Phi JH, Koh EJ, Park WY, Park SH, Hwang DW, et al: miR-192 suppresses leptomeningeal dissemination of medulloblastoma by modulating cell proliferation and anchoring through the regulation of DHFR, integrins, and CD47. Oncotarget 6: 43712-43730, 2015.

39. Zhang J, Li N, Fu J and Zhou W: Long noncoding RNA HOTAIR promotes medulloblastoma growth, migration and invasion by sponging miR-1/miR-206 and targeting YY1. Biomed Pharmacother 124: 109887, 2020.

40. Senfter D, Samadaei M, Mader R, Gojo J, Peyrl A, Krupitza G, Kool M, Sill M, Haberler C, Ricken G, et al: High impact of miRNA-4521 on FOXM1 expression in medulloblastoma. Cell death \& disease 10: 696, 2019.

41. Kumar V, Kumar V, Chaudhary AK, Coulter DW, McGuire T and Mahato RI: Impact of miRNA-mRNA profiling and their correlation on medulloblastoma tumorigenesis. Mol Ther Nucleic Acids 12: 490-503, 2018.

42. Li Y, Jiang T, Shao L, Liu Y, Zheng C, Zhong Y, Zhang J and Chang Q: Mir-449a, a potential diagnostic biomarker for WNT group of medulloblastoma. J Neurooncol 129: 423-431, 2016.

43. Pezuk JA, Brassesco MS, De Oliveira RS, Machado HR, Neder L, Scrideli CA and Tone LG: PLK1-associated microRNAs are correlated with pediatric medulloblastoma prognosis. Childs Nerv Syst 33: 609-615, 2017.

44. de Antonellis P, Medaglia C, Cusanelli E, Andolfo I, Liguori L, De Vita G, Carotenuto M, Bello A, Formiggini F, Galeone A, et al: MiR-34a targeting of Notch ligand delta-like 1 impairs $\mathrm{CD} 5^{+} / \mathrm{CD} 133^{+}$tumor-propagating cells and supports neural differentiation in medulloblastoma. PLoS One 6: e24584, 2011

45. Silber J, Hashizume R, Felix T, Hariono S, Yu M, Berger MS, Huse JT, VandenBerg SR, James CD, Hodgson JG and Gupta N: Expression of miR-124 inhibits growth of medulloblastoma cells. Neuro Oncol 15: 83-90, 2013. 
46. Nigro JM, Cho KR, Fearon ER, Kern SE, Ruppert JM, Oliner JD, Kinzler KW and Vogelstein B: Scrambled exons. Cell 64: 607-613, 1991.

47. Salzman J, Gawad C, Wang PL, Lacayo N and Brown PO: Circular RNAs Are the predominant transcript isoform from hundreds of human genes in diverse cell types. PLoS One 7: e30733, 2012.

48. Li X, Yang L and Chen LL: The biogenesis, functions, and challenges of circular RNAs. Mol Cell 71: 428-442, 2018.

49. Chen LL: The expanding regulatory mechanisms and cellular functions of circular RNAs. Nat Rev Mol Cell Biol 21: 475-490, 2020.

50. Nilsen TW and Graveley BR: Expansion of the eukaryotic proteome by alternative splicing. Nature 463: 457-463, 2010.

51. Moore MJ and Proudfoot NJ: Pre-mRNA processing reaches back to transcription and ahead to translation. Cell 136: 688-700, 2009.

52. Salzman J, Chen RE, Olsen MN, Wang PL and Brown PO Cell-type specific features of circular RNA expression. PLoS Genet 9: e1003777, 2018.

53. Hansen TB, Jensen TI, Clausen BH, Bramsen JB, Finsen B, Damgaard CK and Kjems J: Natural RNA circles function as efficient microRNA sponges. Nature 495: 384-388, 2013.

54. Belter A, Popenda M, Sajek M, Woźniak T, NaskretBarciszewska MZ, Szachniuk M, Jurga S and Barciszewski J: A new molecular mechanism of RNA circularization and the microRNA sponge formation. J Biomol Struct Dyn: Nov 17, 2020 (Epub ahead of print).

55. Guo JU, Agarwal V, Guo H and Bartel DP: Expanded identification and characterization of mammalian circular RNAs. Genome Biol 15: 409, 2014

56. Xie H, Ren X, Xin S, Lan X, Lu G, Lin Y, Yang S, Zeng Z, Liao W, Ding YQ and Liang L: Emerging roles of circRNA_001569 targeting miR-145 in the proliferation and invasion of colorectal cancer. Oncotarget 7: 26680-26691, 2016

57. Wei Y, Chen X, Liang C, Ling Y, Yang X, Ye X, Zhang H, Yang P, Cui X, Ren Y, et al: A noncoding regulatory RNAs Network Driven by Circ-CDYL acts specifically in the early stages hepatocellular carcinoma. Hepatology 71: 130-147, 2020.

58. Han J, Zhao G, Ma X, Dong Q, Zhang H, Wang Y and Cui J: CircRNA circ-BANP-mediated miR-503/LARP1 signaling contributes to lung cancer progression. Biochem Biophys Res Commun 503: 2429-2435, 2018.
59. Gong J, Jiang H, Shu C, Hu MQ, Huang Y, Liu Q, Li RF and Wei YZ: Integrated analysis of circular RNA-associated ceRNA network in cervical cancer: Observational Study. Medicine (Baltimore) 98: e16922, 2019.

60. Huang M, He YR, Liang LC, Huang Q and Zhu ZQ: Circular RNA hsa_circ_0000745 may serve as a diagnostic marker for gastric cancer. World J Gastroenterol 23: 6330-6338, 2017.

61. Li G, Huang M, Cai Y, Yang Y, Sun X and Ke Y: Circ-U2AF1 promotes human glioma via derepressing neuro-oncological ventral antigen 2 by sponging hsa-miR-7-5p. J Cell Physiol 234: 9144-9155, 2019.

62. Lv T, Miao Y, Jin K, Han S, Xu TQ, Qiu ZL and Zhang XH: Dysregulated circular RNAs in medulloblastoma regulate proliferation and growth of tumor cells via host genes. Cancer Med 7: 6147-6157, 2018

63. Wang X, Xu D, Pei X, Zhang Y, Zhang Y, Gu Y and Li Y: CircSKA3 modulates FOXM1 to facilitate cell proliferation, migration, and invasion while confine apoptosis in medulloblastoma via miR-383-5p. Cancer Manag Res 12: 13415-13426, 2020.

64. Shibayama Y,Fanucchi S, Magagula Land Mhlanga MM: IncRNA and gene looping: What's the connection? Transcription 5: e28658, 2014.

65. Lin CY, Erkek S, Tong Y, Yin L, Federation AJ, Zapatka M, Haldipur P, Kawauchi D, Risch T, Warnatz HJ, et al: Active medulloblastoma enhancers reveal subgroup-specific cellular origins. Nature 530: 57-62, 2016.

66. Shi PF, Ji HL, Luo YK, Mao TM, Chen X and Zhou KY: Effect of long noncoding RNA SPRY4-IT1 on proliferation and metastasis of medulloblastoma. Zhongguo Ying Yong Sheng Li Xue Za Zhi 33: 78-82, 2017 (In Chinese).

This work is licensed under a Creative Commons Attribution-NonCommercial-NoDerivatives 4.0 International (CC BY-NC-ND 4.0) License. 